\title{
Frequence Et Facteurs Associes Aux Accouchements Prematures Au Centre Hospitalier Universitaire Et Departemental Du Borgou Alibori Au Benin
}

\author{
Hounkponou N.F.M., \\ Unité d'enseignement et de recherche en Gynécologie-Obstétrique, Faculté \\ de Médecine, Université de Parakou, Bénin
}

\section{Tonato Bagman A.,}

Unité d'enseignement et de recherche en Gynécologie-Obstétrique, Faculté des Science de la Santé d'Abomey Calavi, Bénin

\section{Ahouingnan AY., Gbevo S.,}

Unité d'enseignement et de recherche en Gynécologie-Obstétrique, Faculté de Médecine, Université de Parakou, Bénin

\section{Laourou H.,}

Ecole Nationale de Formation des Techniciens Supérieurs en Sante Publique et en Surveillance Epidémiologique

\section{Vodouhe M.,} Sidi RI, Obossou A., Salifou K.,

Unité d'enseignement et de recherche en Gynécologie-Obstétrique, Faculté de Médecine, Université de Parakou, Bénin

doi: 10.19044/esj.2017.v13n24p427 URL:http://dx.doi.org/10.19044/esj.2017.v13n24p427

\begin{abstract}
Introduction: The premature birth constitutes a major cause of the neonatal and maternal mortality and morbidity and it occurs between 28 weeks of amenorrhoea and 36 weeks plus six days. Objectives: It is to determine the frequency and the factors associated to the premature at the maternity at the Departmental and Teaching Hospital of Borgou. Tool and methodological approaches: It is about an analytical and descriptive retrospective study carried out at the Hospital Teaching Centre of Borgou. The study has been conducted from the $1^{\text {st }}$ January to $30^{\text {th }}$ June 2016. Results: From the 665 retained deliveries, 119 were premature births, be a frequency of $17.89 \%$. The bracket age of 20-34 years was the most represented with $69,75 \%$. The pauci gests and the primipara were
\end{abstract}


respectively in $36.13 \%$ and $40.34 \%$ of the cases. The factors of risk such as premature rupture of membranes (PRM; $\mathrm{p}=0.00001)$, the haemorrhagic prævia placenta $(p=0.0001)$, the multiple pregnancies $(p=0.000003)$ were associated with prematurity. Conclusion: The prematurity constitutes a problem of public health in parakou and is responsible for the heavy consequences on the still-born. Hence, the interest of a better agreement to pay the medical expenses of the factors of risk permitting the reduction of its repercussion.

Keywords: Prematurity, Associated factors, Bénin

\section{Résumé}

Introduction: La prématurité constitue une cause majeure de morbidité et de mortalité maternelle et néonatale et survient entre $28 \mathrm{SA}$ et $36 \mathrm{SA}+6$ jours. Objectifs: Déterminer la fréquence et les facteurs associés à l'accouchement prématuré à la maternité du Centre Hospitalier Universitaire et Départemental (CHUD) du Borgou. Matériel et méthodes: Il s'agissait d'une étude rétrospective descriptive et analytique effectuée à la maternité du CHUD Borgou. L'étude s'est déroulée du $1^{\text {er }}$ Janvier au 30 Juin 2016. Résultats: Sur 665 accouchements retenus, 119 étaient prématurés, soit une fréquence de $17,89 \%$. Les pauci gestes et les primipares étaient représentés respectivement dans $36,13 \%$ et $40,34 \%$ des cas. La tranche d'âge de 20-34 ans était la plus représentée avec $69,75 \%$. Les facteurs de risque tels que la rupture prématurée des membranes $(\mathrm{RPM} ; \mathrm{p}=0,00001)$, le placenta prævia hémorragique $(p=0,0001)$, les grossesses multiples $(p=0,000003)$ étaient associés à la prématurité. Conclusion : La prématurité constitue un problème de santé publique à la maternité du Centre Hospitalier Départemental et Universitaire (CHUD) de Parakou et est responsable de lourdes conséquences sur les nouveau-nés. D'où l'intérêt d'une meilleure prise en charge de ses facteurs de risque permettant la réduction de son incidence.

Mots clés: Prématurité, Facteurs associés, Bénin

\section{Introduction}

La prématurité constitue le problème le plus important de la pratique obstétricale selon Mahesh (Mahesh et al., 2013). L'accouchement prématuré (AP) se réfère à toute naissance qui se produit entre 28 semaine d'aménorrhée (SA) et 36 SA + 6 jours (Nuzhat et al., 2011). L'AP a un impact très sérieux sur l'augmentation de la mortalité et de la morbidité périnatale aussi bien dans les pays en développement que dans les pays développés (Nuzhat et al., 2011). Son incidence varie entre de 5\% à 15\% (Elvira et al., 2013). Selon l'OMS, environ 15 millions de bébés naissent 
prématurément chaque année dont plus d'un million de décès sont enregistrés (OMS, 2012). La prématurité est la principale cause de décès chez les nouveau-nés (au cours des quatre premières semaines de vie) et la deuxième cause majeure de décès après la pneumonie, chez les enfants de moins de 5 ans (OMS, 2012 ; Stacy et al., 2010). Les naissances prématurées (NP) sont responsables de $75 \%$ de la mortalité néonatale, $50 \%$ de la déficience neurologique à long terme chez les enfants. L'incidence de la prématurité a augmenté au cours des dernières années. Plusieurs facteurs y ont contribué (Farhin et al., 2014). Selon la littérature, les grandes causes de la prématurité sont : les causes fœtales : la souffrance fœtale, les grossesses multiples, le placenta prævia; les causes utérines : anomalies de l'utérus et du col incompétent; causes maternelles: la pré-éclampsie; les maladies chroniques ; les infections (Elvira et al., 2013 ; OMS, 2012). On retrouve d'autres causes telles que : la rupture prématurée des membranes (RPM), le poly-hydramnios (Elvira et al., 2013). Malgré la connaissance de ses multiples causes, une grande partie de son étiologie reste inexpliquée (Dolan et al., 2013). Cette étude vise à déterminer les facteurs associés à l'accouchement prématuré pouvant mieux expliquer sa survenue.

\section{Materiel Et Methodes}

Il s'agissait d'une étude rétrospective descriptive et analytique qui s'est déroulée du $1^{\text {er }}$ Janvier au 30 Juin 2016 à la maternité du Centre Hospitalier Universitaire et Départemental (CHUD) du Borgou-Alibori. Etaient incluses, toutes les patientes ayant accouché à partir de la $28^{\text {ème }} \mathrm{SA}$. Celles ayant accouché d'un mort-né n'ont pas été incluses et celles dont les dossiers sont incomplets nt été exclues. Les variables étudiées étaient: âge de la mère, le niveau d'instruction, les antécédents (ATCD) obstétricaux et gynécologiques, la présence des pathologies au cours de la grossesse, les facteurs étiologiques tels que la RPM, le placenta prævia. La technique de collecte était le dépouillement des dossiers médicaux. Une fiche de dépouillement a été conçue et utilisée à cet effet. Les logiciels EPI data et Epi-Info 7 avaient servi pour la saisie, le traitement et l'analyse des données. Les tests de khi2 ont été utilisés pour la comparaison des fréquences. Tout résultat était significatif pour un $\mathrm{p}<0,05$.

\section{Resultats}

Sur 665 accouchements récencés, 119 étaient prématurés, soit une fréquence de $17,89 \%$. Dans $77,31 \%$ des cas, les prématurés étaient nés entre $33 \mathrm{SA}$ et $36 \mathrm{SA}+6$ jours. La tranche d'âge de 20 à 34 ans était représentée dans $69,75 \%$ des cas. La moyenne d'âge était de 26,70 ans $\pm 6,77$ ans avec des extrêmes de 14 et 43 ans. 
Les ménagères $(22,69 \%)$, les artisanes $(26,05 \%)$, les vendeuses/revendeuses $(18,49)$, les fonctionnaires $(14,29 \%)$, les élèves/étudiantes $(10,08 \%)$ étaient les principales professions retrouvées.

Les parturientes étaient référées dans 63,03\% (75/119) des cas. Les primipares représentaient $40,34 \%$ des cas et les grandes multipares définies comme ayant déjà accouché plus de 5 fois, représentaient $6,72 \%$ des parturientes. Les parturientes étaient des primigestes ( $1{ }^{\text {ère }}$ grossesse $33,61 \%$ ) pauci gestes (2 à 3 grossesses 36,13\%), multi gestes (4 à 5 grossesses $23,53 \%$ ) et des grandes multi gestes (plus de 5 grossesses 6,72\%), Les patientes (23/119) soit 19,33\% avaient un antécédent de fausse couche spontanée, 2 soit $1,68 \%$ de fausse couche provoquée et 20 soit 20,17\% de césarienne. Les antécédents d'hypertension artérielle, de diabète étaient notés dans respectivement $3,36 \%$ et $0,84 \%$ des cas. La consultation prénatale (CPN) avaient été faite au moins trois fois dans $34,45 \%$ des cas et 6 soit $5,04 \%$ n'avaient fait aucune CPN. Il n'y avait aucune relation statistiquement significative entre la prématurité et l'âge de la mère $(p=0,38)$, la profession $(p=0,06)$, les antécédents de fausse couche $(p=0,72)$, la parité $(p=0,37)$ et la gestité $(p=0,50)$. La fréquence de la prématurité était plus élevée dans le groupe des patientes qui présentaient une RPM que dans le groupe des patientes ne présentant pas une RPM (38,81\% versus 15,55\%). Cette différence était statistiquement significative $(p=0,00001)$. Il en de même pour certains facteurs de risque tels que: le placenta prævia hémorragique, le paludisme, la pré-éclampsie, la grossesse gémellaire, la menace d'accouchement prématuré qui étaient tous associés à la survenue de l'accouchement prématuré dans la présente étude. (Tableau I)

Tableau I: Relation entre les facteurs de risque et l'accouchement prématuré, CHUD

Borgou.

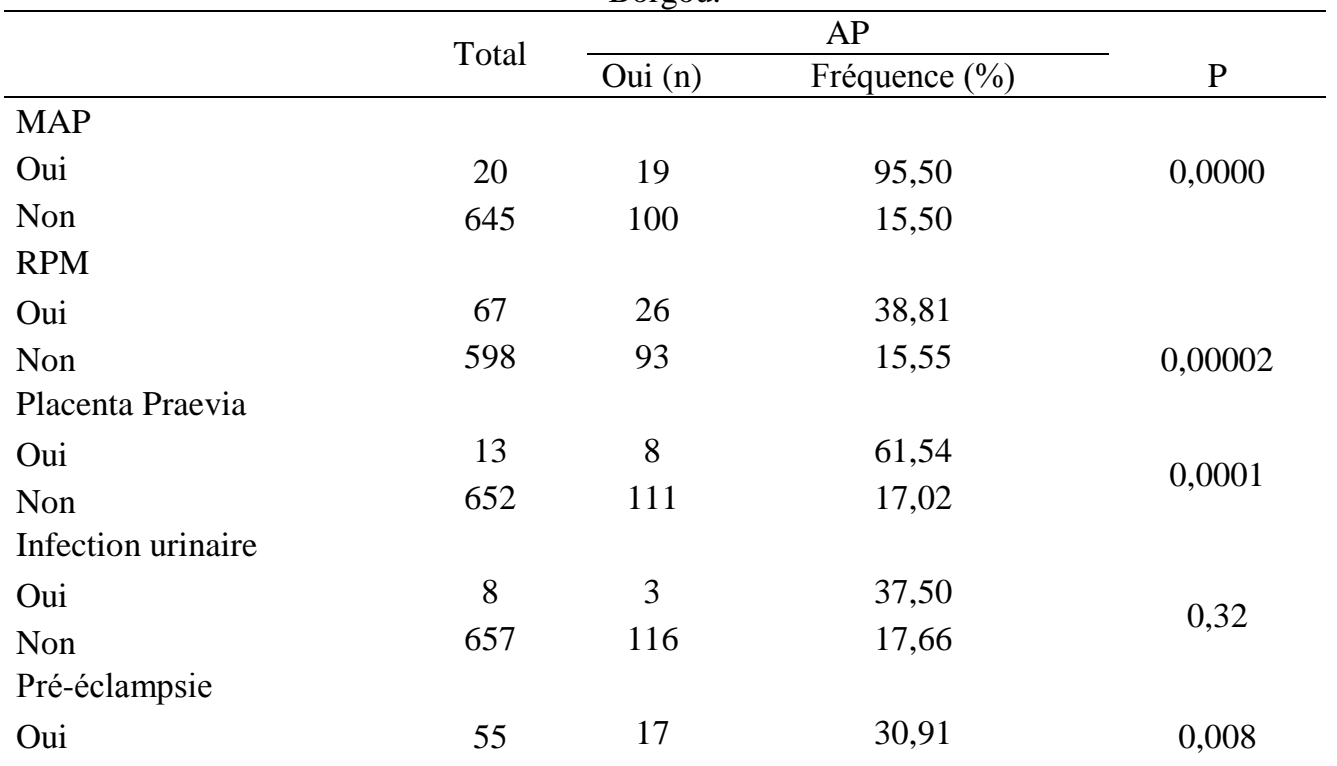




\begin{tabular}{lcccc} 
Non & 610 & 102 & 16,72 & \\
$\begin{array}{l}\text { Paludisme } \\
\text { Oui }\end{array}$ & 10 & 5 & 50,00 & 0,02 \\
Non & 655 & 114 & 17,40 & \\
$\quad$ Grossesse multiple & & & & \\
mono fotale & 632 & 103 & 16,30 & 0,000003 \\
Gémellaire & 33 & 16 & 48,48 & \\
\hline
\end{tabular}

\section{Discussion}

Les naissances prématurées représentent $11,1 \%$ des naissances vivantes dans le monde, dont $60 \%$ proviennent de l'Asie du Sud et de l'Afrique subsaharienne. Dans les pays les plus pauvres, on compte en moyenne $12 \%$ de bébés nés prématurément, comparativement à $9 \%$ dans les pays à revenu plus élevé (OMS, 2012 ; Blencowe et al). Ce qui démontre que la prématurité n'est pas que l'apanage des pays sous développées. Dans cette étude, la fréquence de l'accouchement prématuré était de 17,89\%. Ce taux est beaucoup plus proche de celui trouvé par Farhin et al (Farhin et al., 2014) qui était de $17 \%$, une étude rétrospective effectuée à l'hôpital Dhiraj et de celle trouvée par Pambou et al (Pambou et al., 2006) qui avaient trouvé une fréquence de 16,7\% au CHU de Brazzaville mais inférieure aux 43\% trouvés par Nyenga et al (Nyenga et al., 2013) à l'hôpital sendwe /Lubumbashi en République Démocratique du Congo. Cette fréquence élevée constatée dans l'étude de Nyenga et al pourrait-être expliquée par le fait que l'étude du Congo est réalisée dans un service de néonatologie qui est un service de référence recevant des nouveau-nés de plusieurs maternités. Les auteurs avaient donc tenu compte aussi bien des patientes ayant accouché à l'hôpital de Sendwe que des patientes ayant accouché dans d'autres centres de santé et dont les enfants étaient pris en charge au service de néonatologie de cet hôpital alors que notre étude menée a pris en compte que des patientes ayant accouché uniquement à la maternité du CHUD Borgou. La prévalence de la prématurité tournerait entre 7\% et 16\% (Mahesh et al., 2013). Les données récentes sur les naissances prématurées au Bénin sont rares. Toutes fois selon un rapport de l'OMS publié en 2012, le taux de prématurité au Bénin en 2010 était estimé à 10,6\% (OMS, 2012). Ce taux est confirmé dans une étude de Blencowe et al en 2012. Toujours selon ce rapport, sur les onze (11) pays qui présentent un taux de prématurité supérieur à $15 \%$, neuf (9) proviennent de l'Afrique Subsaharienne (OMS, 2012 ; Blencowe et al., 2012) (Tableau II).

Tableau II: Liste des pays détenant un taux de prématurité supérieur à 15\%, OMS, 2012.

\begin{tabular}{lll}
\hline Pays & $\begin{array}{l}\text { Taux de naissances } \\
\text { naissances }(2010)\end{array}$ & \\
\hline Malawi & & 18,1 \\
Congo & 16,7
\end{tabular}




$\begin{array}{ll}\text { Comores } & 16,7 \\ \text { Zimbabwe } & 16,6 \\ \text { Guinée équatoriale } & 16,5 \\ \text { Mozambique } & 16,4 \\ \text { Gabon } & 16,3 \\ \text { Pakistan } & 15,8 \\ \text { Indonésie } & 15,5 \\ \text { Mauritanie } & 15,4 \\ \text { Botswana } & 15,1\end{array}$

16,7

16,6

16,5

16,4

16,3

15,8

15,5

15,1

La fréquence de 17,89\% trouvée dans notre étude pourrait aussi s'expliquer par le fait que les accouchements normaux sont pris en charge dans les centres de santé périphériques et les accouchements difficiles comme les accouchements prématurés sont transférés au CHUD Borgou qui est la structure de référence au niveau départemental.

Dans cette étude, l'âge gestationnel moyen chez les patientes ayant accouché de façon prématurée était de 34,04 SA. La grande majorité des prématurés était née entre $33 \mathrm{SA}$ et $36 \mathrm{SA}+6$ jours dans $77,31 \%$ des cas. Pour cette même tranche, Nyenga et al (Nyenga et al., 2013) avaient trouvé 62\%. Dans l'étude de Farhin et al (Farhin et al., 2014), 46\% des patientes avaient accouché entre $32 \mathrm{SA}$ et $36 \mathrm{SA}$. Trois classes majeures existent quant à la répartition des prématurés (OMS, 2012) : la prématurité moyenne (32$36 \mathrm{SA}+6$ jours); la grande prématurité ( $28 \mathrm{SA}$ et $31 \mathrm{SA}+6$ jours); l'extrême prématurité (avant $28 \mathrm{SA}$ ). La tranche d'âge la plus représentée dans notre étude correspondait à celle de 20 à 34 ans dans $69,75 \%$ des cas. Chiabi et al (Chiabi et al., 2013) rapportaient $66,94 \%$ pour cette même tranche dans une étude réalisée au Cameroun. Dans notre étude, l'accouchement prématuré a été retrouvé chez l'adolescente ( $\leq 19$ ans) et chez la femme âgée ( $\geq 35$ ans) dans respectivement $11,73 \%$ et $13,98 \%$ des cas. Elvira et al (Elivra et al., 2013) dans leur étude ont trouvé une incidence d'accouchement prématuré de $4,4 \%$ chez les patientes de moins de 18 ans et une incidence de $14 \%$ chez les femmes de plus de 35 ans. Aux Etats-Unis, le taux de naissance prématurée chez les femmes âgées de 20 à 35 ans se situait entre 11 à 12\%; alors qu'il atteignait plus de $15 \%$ chez les femmes de moins de 17 ans et de plus de 40 ans (OMS, 2012). La prématurité se produit plus souvent chez les femmes enceintes de moins de 18 ans et plus de 35 ans (Elivra et al., 2013) . Mais dans cette étude, l'âge n'était pas associé à la survenue de l'AP $(\mathrm{p}=0,38)$.

Aucun antécédent de prématurité n'a été noté chez aucune des patientes mais il est signalé qu'un AP antérieur constituait un risque élevé de conduire à une naissance prématurée (Dolan et al., 2013). Par contre l'antécédent de fausse couche spontanée avait été retrouvé dans 19,33\% des cas dans l'actuelle étude. 
Six (6) patientes n'avaient fait aucune consultation prénatale (CPN) dans la présente étude soit $5,04 \%$. Cela pourrait s'expliquer par l'ignorance des patientes par rapport à l'utilité des $\mathrm{CPN}$, les grossesses non désirées et voire le manque de moyens financiers. Aussi $34,45 \%$ des patientes avaient moins de trois (3) CPN. Ndiaye et al. en 2006 ont démontré que le non suivi ou le mauvais suivi de la grossesse contribuait à la survenue des accouchement prématuré et avaient indiqué qu'un nombre de CPN inférieur à 3 avait un risque significativement élevé d'accouchement prématuré alors qu'un nombre de $\mathrm{CPN}=3$ avait un effet protecteur. Certaines pathologies maternelles rencontrées et citées comme facteurs de risque de l'accouchement prématuré (AP) dans la littérature ne se sont pas confirmées dans cette étude. L'infection urinaire désignée par Mokuolu et al 2014 comme un facteur de risque de l'AP n'a pas été constatée dans notre étude.

Néanmoins, parmi les huit patientes qui présentaient une infection urinaire, trois patientes avaient accouché de façon prématurée. Le paludisme était significativement associé à l'accouchement prématuré $(\mathrm{p}=0,02)$. Ndiaye et al en 2006 étaient parvenus à la même conclusion et avaient noté que le paludisme était le seul facteur pathologique associé à la prématurité. Le paludisme, maladie parasitaire fébrile pouvant provoquer des contractions utérines. La RPM a une relation étroite avec la prématurité. Il est responsable de près de $30 \%$ de toutes les naissances prématurées (Guinsburg et al., 2010). Selon le Guinsburg et al., 2010, la RPM était présente chez 21,85\% des patientes qui avaient accouché prématurément et la fréquence de l'accouchement prématuré était plus élevée dans le groupe des patientes présentant une RPM que des patientes ne présentant pas une RPM $(38,81 \%$ versus $15,55 \%)$. La RPM était statistiquement associée à l'AP dans notre étude ( $\mathrm{p}=0,00001)$. Il en est de même pour les facteurs tels que les grossesses multiples (grossesse gémellaire dans cette étude). L'AP était plus élevé au niveau des grossesses gémellaires que chez les grossesses mono fœtales $(48,48 \%$ versus $16,30 \%)$ avec une différence statistiquement significative ( $p=0,000003$ ). Mahesh et al en 2013 confirment le fait que les grossesses multiples constituent un facteur de risque de la prématurité. Guinsburg et al 2010 ont également prouvé qu'il existe une forte relation entre la prématurité et les grossesses multiples et l'une des raisons évoquées de cette association est le début et l'étirement exagéré des fibres myométriales. La pré-éclampsie était associée à la prématurité dans l'étude $(\mathrm{p}=0,008)$. Par contre, Ndiaye et al en 2006 n'avaient trouvé aucune association entre l'AP et la prééclampsie. Le placenta prævia était un facteur de risque de l'AP dans cette étude. Près de $62 \%$ des patientes avec un placenta prævia, avaient accouché de façon prématurée contre $17,02 \%$ chez les patientes ne présentant pas un placenta prævia. La différence était statistiquement significative $(\mathrm{p}=0,0001)$. 
La Menace d'Accouchement Prématuré (MAP) était significativement associée à l'AP dans notre étude. La majorité (95\%) des patientes présentant une MAP, avait accouché prématurément. Ce taux élevé pourrait s'expliquer par la non prise en charge à temps de ces cas de MAP. Il importe donc d'éviter la prématurité par la prise en charge adéquate des MAP.

\section{Conclusion}

La prématurité est une urgence obstétricale qu'il convient de prévenir au mieux. Sa fréquence est de 17,89\% dans cette étude. Des facteurs de risque ont été associés à la survenue des accouchements prématurés. L'action préventive doit être la prévention primaire des facteurs associés, en termes cliniques des facteurs étiologiques.

\section{References:}

1. Mahesh R, Asalkar, Pradeep R, Gaikwad, Ritesh P. Perinatal morbidity and mortality due to preterm deliveries in a referral hospital, in rural India: a cross sectional study. Int J Reprod Cont, Obstet Gynecol 2013, 2:555-61.

2. Nuzhat R, Iffat Y, Sumaira S. Role of Nifedipine in comparaison to salbutamol in the management of preterm labour. PJMHS 2011, 5(1): 63-6.

3. Elvira B, Gordana G, Dženita L, Edin O, Azur T. etiological factors of preterm delivery. J Health Scie 2013, 3: 159-63.

4. Organisation Mondiale de la Santé (OMS). Arrivés trop tôt : rapport des efforts mondiaux sur les naissances prématurées; Mai 2012. [Disponible en ligne] : www.who.int/pmnch/media/news/2012/preterm_birth_report/en/inde x.html.

5. Stacy B, Daniel W, Lales S, Ana PB, Mario M, Jennifer HR et al. incidence mondiale de la naissance avant terme: revue systématique de la mortalité et de la morbidité maternelle. Bull OMS 2010,88: 80p.

6. Farhin R, Deepak AD, Jyoti S, Pawanpreet K. preterm birth and its outcome. Int J Reprod Contracept Obstet Gynecol 2014, 3: 153-7.

7. Dolan SM, Christiaens I. Genome-wide association studies in preterm birth: implications for the practicing obstetrician-gynaecologist. BMC pregnancy and Chilbirth 2013, 13. [Disponible en ligne]: http://www.biomedcentral.com/1471-2393/13/S1/S4. 
8. Blencowe H, Cousens S, Oestergaard M, Chou D, Moller AB, Nawal $\mathrm{R}$ et al. National regional and worldwide estimates of preterm birth. The Lancet 2012, 379: 2162-72.

9. Pambou O, Ntsika-kaya P, Ekoundzola JR, Mayanda F. Naissances avant terme au CHU de Brazzaville. JL Eurotext 2006, 16(3): 185-9.

10. Nyenga MA, Mwananteba A, Kanteng AW, Lubala KT, Yaba A. Profil et risque de mortalité chez les prématurés à l'hôpital Sendwe/Lubumbaki, RD Congo. Rev Ped Fleuv Congo 2013, 1(3): 16.

11. Chiabi A, Evelyn MM, Nicole M, seraphin N, Lawrence M, Karen KK et al. Risk factors for premature births : a cross-sectional analysis of hospital recors in a cameroonian health facility. Afr J Reprod Health, 2013; 17(4): 77-83.

12. Ndiaye O, Fall AL, Dramé A, Sylla A, Guèye M, Cissé CT et al. Facteurs étiologiques de la prématurité au centre hospitalier régional de Ziguinchor. Bull Soc Pathol Exot, 2006 ; 16(3): 185-9.

13. Mokuolu OA, Suluman BM, Adesiyum OO, Adéniyi A. prévalence et déterminants des livraisons anticipées au centre hospitalier universitaire de Iloris, Nigéria. Ped Reprod 2014, 3(2): 442-6.

14. Guinsburg R, Cecatti JG, Marba ST, Tedesco RP, Passini R, Martinez FE et al. Brazilian multicenter study on prevalence of preterm birth and associated factors. BMC Pregnancy and Childbirth 2010, (10). [En ligne]: http://www.biomedcentral.com/1471$\underline{2393 / 10 / 22}$ 